\title{
Current formulation approaches in design and development of solid oral dosage forms through three-dimensional printing
}

\author{
Carlo Curti $^{1}$ D . Daniel J. Kirby ${ }^{1}$ D $\cdot$ Craig A. Russell $^{1}$ (D)
}

Received: 15 August 2019 / Accepted: 6 March 2020 / Published online: 21 March 2020

(c) The Author(s) 2020

\begin{abstract}
Three-dimensional (3D) printing technologies are continuously applied to novel fields, laying the foundations for a new industrial revolution. With regard to pharmaceutical sciences, 3D printed drug products are emerging as attractive and innovative tools in personalised medicine. For example, solid oral dosage forms (e.g. tablets) can be printed in a wide range of dosages, release profiles, geometries and sizes by simply modifying a digital model, thus providing patients with tailored therapies. Various 3D printing technologies have been applied to pharmaceutical manufacture in recent years, and different materials have been investigated to fabricate solid oral dosage forms in a broad range of properties. Therefore, the aim of this review is to describe the state of the art of 3D printing oral pharmaceuticals, with the view to provide formulation scientists with essential information to approach the development of 3D printed drug products, from digital design to final product quality control. Short- to long-term potential areas of application of 3D printed drug products and their relative regulatory pathway challenges are also presented.
\end{abstract}

Keywords Drug delivery $\cdot$ Personalised medicine $\cdot$ Three-dimensional printing $\cdot$ Solid oral dosage forms

\section{Introduction}

Automated production of solid oral dosage forms was introduced over two centuries ago [1]. The main advantages of such dosage forms are based on their relatively undemanding and convenient manufacture, coupled with high patient compliance. Tablets, the most relevant exponent of the class, have been improved in the last decades introducing techniques such as film coating, double compression and osmotic systems to achieve controlled and targeted release. However, despite the technological advances, tablet production is still based on tableting machines whose design has not essentially changed for decades since the introduction of automated tablet presses [2]. Conventional tableting techniques still suffer from limitations, including challenges in direct compression of powders and the related need for granulation. A further drawback of conventional tableting relies on the increasing demand for personalised treatments, likely to represent a significant shift in future medicine [3, 4]. Indeed,

Craig A. Russell

c.russell6@aston.ac.uk

1 School of Pharmacy, Aston University, Aston Triangle, Birmingham B4 7ET, UK conventional tableting machines based on punches and dies are designed for a mass production market, therefore they are inherently lacking in flexibility; hence, a more flexible platform would facilitate the design, production and dispensing of bespoke medicines in the near future [5].

Additive manufacturing (AM), a set of techniques including $3 \mathrm{D}$ printing, has recently aroused much interest in pharmaceutics due to its large flexibility (Fig. 1), which makes it a promising tool to produce the bespoke drug delivery devices, including solid oral dosage forms [6].

\section{Designing 3D printed solid oral dosage forms: from digital design to bespoke tablet properties}

\subsection{Computer-aided design tools for dosage form design}

Prior to 3D printing solid oral dosage forms, it is essential to produce a digital model through computer-aided design (CAD) software. Digital modelling allows freedom of design such that the production of tablets, for instance, is no longer dependent on size and shape of dies and punches, eventually 


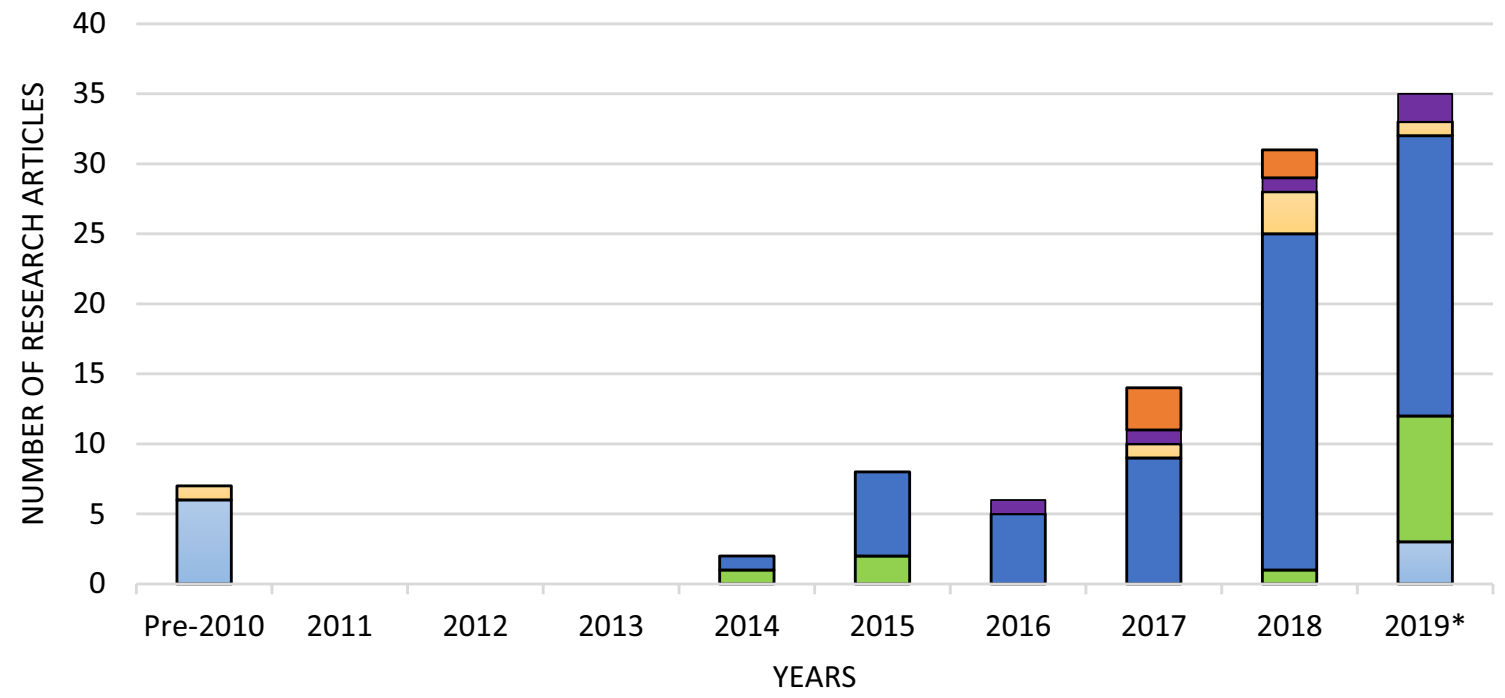

\author{
$\square$ Powder Bed-Inkjet 3DP \\ $\square$ Fused Deposition Modeling (FDM) \\ $\square$ Stereolithography (SLA)
}

Fig. 1 Number of research articles related to 3D printing of solid oral dosage forms published within the years 1996-2010 (grouped as pre-2010) to 2019 (partial data updated to June 2019). Coloured bars represent different 3D printing technologies. Google scholar, Science

\section{$\square$ Pressure Assisted Microsyringe (PAM) \\ $\square$ Selective Laser Sintering (SLS) \\ 口UV or Hot Melt Inkjet 3DP}

direct and PubMed search engines were used to find relevant peerreviewed research articles. Key words for searching were '3D printing', 'solid oral dosage forms', 'tablets' and 'personalised medicine'

dosage forms with different shapes with the aim of assessing the feasibility of SLA 3D printing for future research (Fig. 2). making the same equipment suitable for producing an unlimited variety of geometries. For example, our research group has used Tinkercad (Autodesk Inc., USA) to design a set of
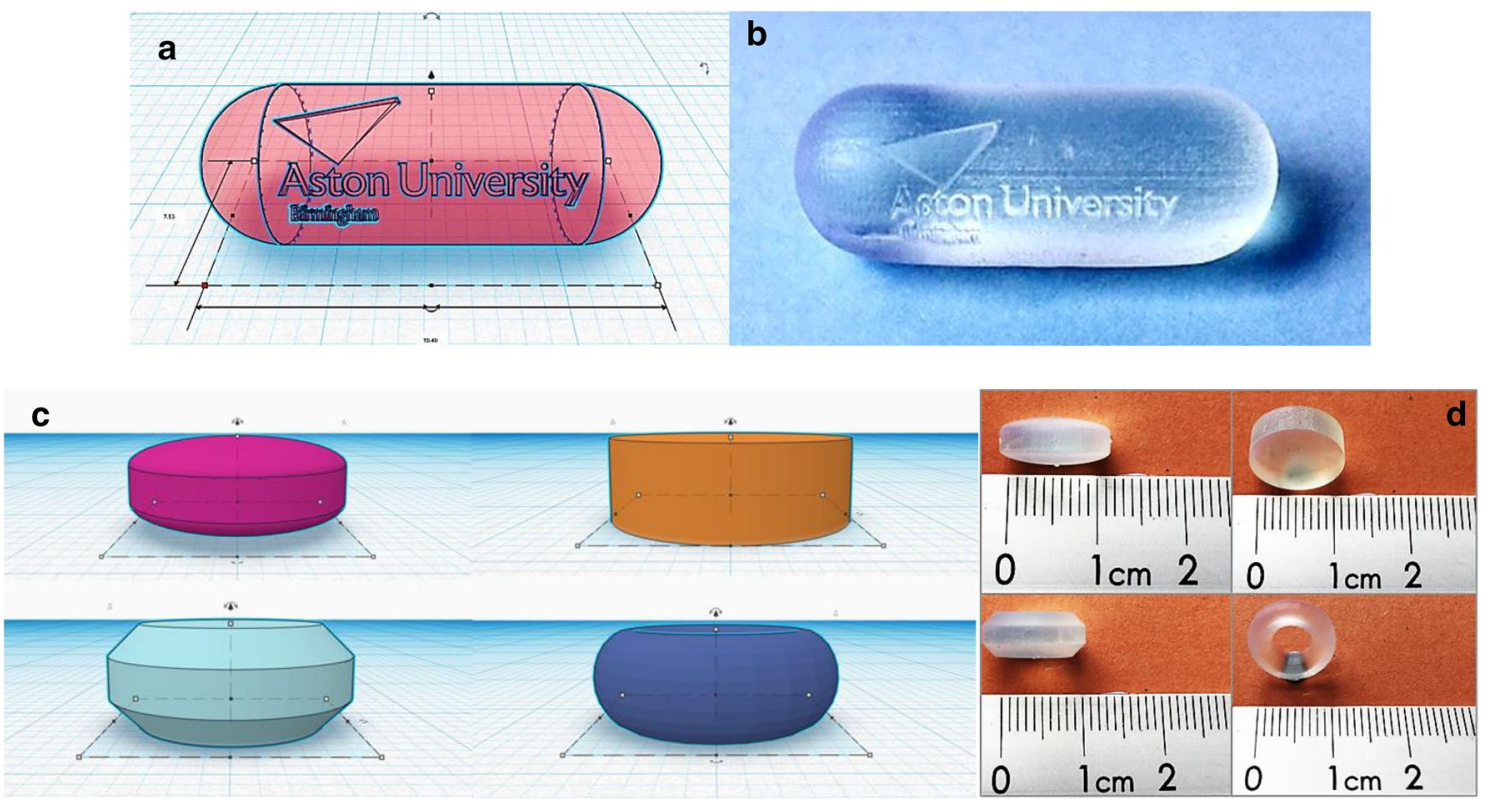

Fig. 2 a, c CAD models of drug delivery devices designed on Tinkercad (Autodesk Inc, USA) and b, d 3D printed prototypes based on the same concept fabricated with a Form 2 SLA 3D printer (FormLabs Inc, USA) using a commercial resin 


\subsection{Tuning dosage form properties through digital design}

Once it is administered to the human body, a solid oral dosage form undertakes a sequence of steps that eventually allows the active pharmaceutical ingredients (API) to be absorbed through the biological membranes and pass to the bloodstream. Tablet disintegration and dissolution play a key role in such process and they are dependent upon a wide range of parameters.

While the type of AM technology used mainly affects tablet disintegration, dissolution time is influenced by the formulation composition and by tablet geometry.

Conventional tablet shapes include round, square, rectangle, capsule and oval; however, more complex geometries are limited by the nature of the compression process itself. In contrast, AM allows the formulation scientist to

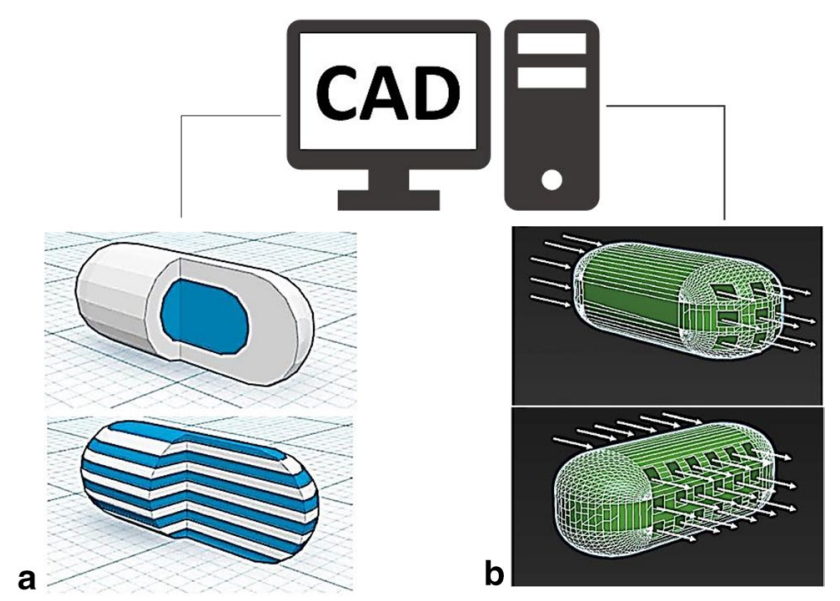

Fig. 3 Schematic diagram representing examples of two different approaches to tune dosage forms' properties through CAD design: a multi-material 3D printing and $\mathbf{b}$ engineering strategies (e.g. channelled tablets). Picture readapted with permission from $[10,15]$ introduce tablet design as a novel stage in the development of more sophisticated products.

Tuning dosage form properties through digital design relies on either fabricating devices where different excipients and/or APIs are deposited in specific regions (multimaterial 3D printing), or engineering approaches (Fig. 3). Multi-material 3D printing of tablets includes several examples, like multilayer polypills and composite tablets, where different layers may consist of different polymers to control dissolution behaviour, or liquid-filled capsules [7-9]; a further relevant example of design-driven drug release mechanisms is subsequently shown in Fig. 4.

Engineering approaches are instead used to modify tablet properties by simply varying their geometries, with no need to change the formulation. For instance, channelled tablets show an accelerated drug release due to the increased surface area to volume ratio [10], while a multi-block tablet design results in a quicker fragmentation compared to no-gaps devices, thus avoiding the need for a disintegrant [11]. A further example is altering tablet density by varying the infill percentage in fused deposition modelling (FDM) 3D printed tablets; as a result, different drug release profiles can be achieved without modifying the formulation $[12,13]$. The advantages of such approach are significative, since dosage forms tailored on the needs of single patients could be manufactured at a moment using the same equipment and feedstock material, with a positive outcome on treatments costs.

Finally, novel dosage form design could also be used to develop abuse deterrent formulations (ADFs); egg-shaped tablets were recently described as crush resistant, showing a force required $>500 \mathrm{~N}$ and, therefore, they could inhibit abusers' manipulation in the form of snorting, inhaling and chewing [14].
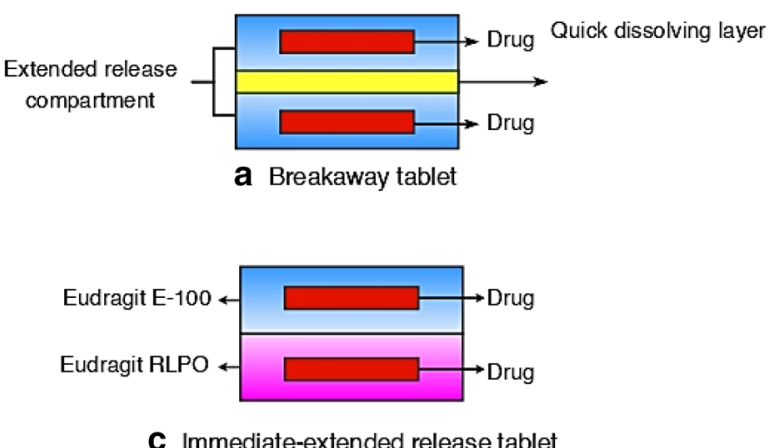

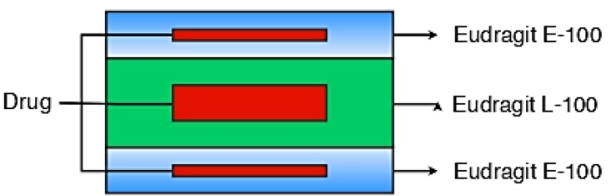

b Dual pulsatory release tablet

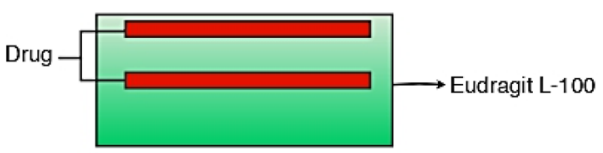

d Enteric dual pulsatory release tablet

Fig. 4 Drug delivery devices designed and fabricated by Rowe et al. a breakaway tablet, $\mathbf{b}$ pulsatory release tablet, $\mathbf{c}$ immediate-extended release device and $\mathbf{d}$ enteric dual pulse release tablet. Picture adapted from [19] 


\section{Applications of additive manufacturing in solid oral dosage form development}

\subsection{Powder bed-inkjet 3D printing}

In inkjet three-dimensional printing, objects are fabricated by spreading a thin layer of a powdered material which is then bound in selected regions by printing a binder solution using an inkjet-type nozzle. Once bound, the powder bed is lowered by a piston and the process is repeated until the whole part is produced. From a pharmaceutical perspective, such a process implies the formulation of both a powder mixture and a liquid ink, where the latter represents the most challenging step due to potential nozzle clogging issues [16]. The API can be either solubilised in the liquid binder or blended with the powder mixture. When the drug is dissolved in the liquid binder, it can be loaded at very low concentrations in the tablets; this would benefit the formulation of drug products requiring ultralow dosages (e.g. hormones). When the API is instead dispersed in the powder blend, the quantity of necessary excipients reduces because of the fewer formulation issues to overcome.

Three-dimensional printing potential in drug delivery was first explored in 1996 at the MIT, where a desktop 3D printer (developed at the MIT itself) was used to produce a device made of polymeric material featuring internal cells [17]. The ability of controlling drug release profiles was demonstrated by depositing dyes only in selected cells, thus introducing a new feature in solid dosage form design and development: microstructural control.

Following this proof of concept, 3D printed dosage forms with both erosion and diffusion drug release mechanism were realised by using specific tablets' design and conventional pharmaceutical excipients [18].

The introduction of more sophisticated technologies, such as the TheriForm ${ }^{\mathrm{TM}}$ process, also made it possible to tune tablet properties through spatial control of composition. Immediate-extended release, breakaway, enteric dual pulsatory and dual pulsatory tablets are examples of dosage forms produced following digital design modelling
(Fig. 4), highlighting a flexibility which is unachievable with conventional tablet pressing techniques [19].

Another appealing application of powder-bed 3D printing is designing zero-order drug release devices [20]. Zero-order release kinetics, defined as constant drug release over time, is a desirable attribute for oral formulations containing APIs with short half-life or narrow therapeutic index, because this allows for minimum fluctuations in drug plasma levels [21, 22]. Reported examples of constant release $3 \mathrm{D}$ printed tablets include devices made of a top and bottom layer impermeable to water to limit drug release from the sides of the tablets, and a middle compartment formed by annular sections with a porosity gradient achieved by printing a lower amount of binder solution (Fig. 5) [20]. The porosity gradient was exploited to have a stable erosion volume, thus resulting in a linear drug release.

Another strategy to achieve linear drug release can be based on torus-shaped tablets, designed to release drug molecules only from the outer and inner surfaces [23].

Powder-bed 3D printing can be furthermore employed to formulate fast-disintegrating tablets, achieved by binding powdered material in a gradient fashion, resulting in a final tablet with inner compartments of loose powder and a highly porous structure (Fig. 6) [24].

High porosity also characterises Spritam ${ }^{\circledR}$, the first medicine fabricated through a $3 \mathrm{D}$ printing process-known as ZipDose ${ }^{\circledR}$ - to gain Food and Drug Administration approval in 2015, marketed by Aprecia Pharmaceuticals [25]. Spri$\operatorname{tam} \AA$ is an antiseizure drug containing the active ingredient levetiracetam and the rationale behind its development is that the highly porous structure allows the tablet to disintegrate in a mean of $11 \mathrm{~s}$, allowing a fast onset of action [26, 27].

Although powder-bed 3D printing allowed important proof of concept studies in 3D printing pharmaceuticals, demonstrating flexibility of the technique as well as the feasibility of using conventional excipients, its application remains poor. Limitations mainly attributable to the use of powders, such as low powder flowability, influence both the process and the final product (Prescott and Barnum, 2000). A low powder flowability can cause the deposition of nonuniform layers, thus affecting dosing homogeneity [16]. Furthermore, the use of powders affects the resolution of
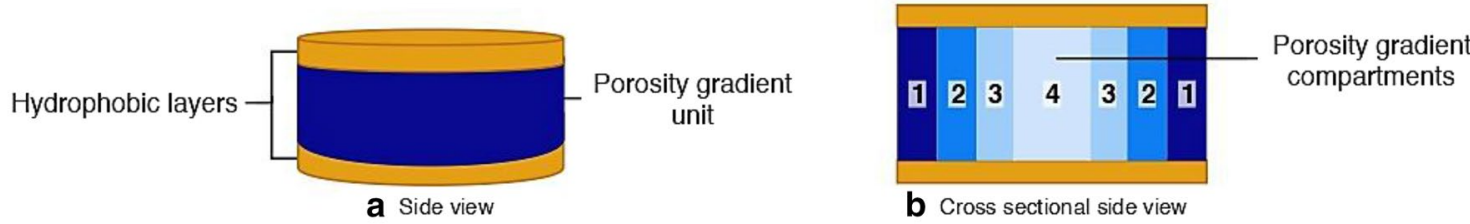

Fig. 5 Schematic representation of a material gradients tablet. A different porosity degree allows a faster erosion when the surface area decreases resulting in a constant drug release Picture adapted from [20] 


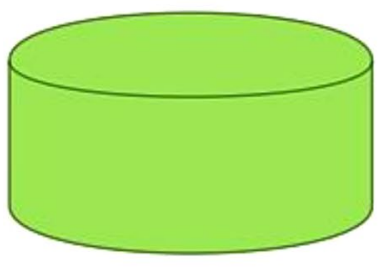

a Side view

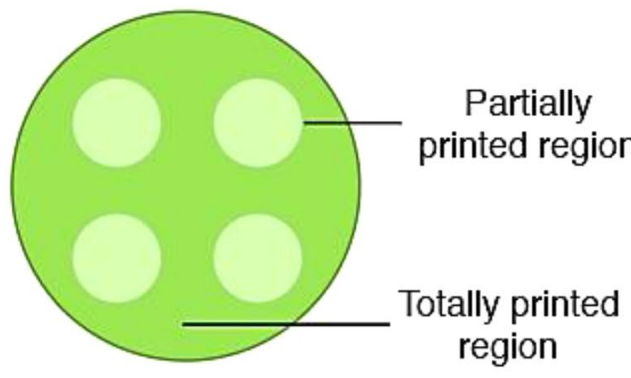

b Cross sectional top view

Fig. 6 Schematic representation of a fast disintegrating tablet. Partially printed regions consist in loose powder that allows faster disintegration and dissolution. Picture adapted from [24]

printed tablets, usually resulting in a rough surface finishing, which might require coating processes to gain a satisfactory patient acceptability. Lastly, despite the valid applications previously reported, highly porous structures showed high friability that could not meet the pharmacopoeia requirements [16, 24, 28].

\subsection{Pressure-assisted microsyringe 3D printing}

Pressure-assisted microsyringe 3D printing (PAM) was introduced in 2006 and represented a breakthrough point in the accessibility of affordable multi-material 3D printers [29]. PAM 3D printers are equipped with one or multiple syringes that can deposit a variety of materials, including liquids, gels or paste.

As different materials can be printed at the same time, the first attempt to manufacture oral dosage forms using such technology describes the formulation of bilayer tablets using gels consisting of suitable excipients to obtain immediate and sustained release layers [30].

More complex PAM 3D printers also allows the production of 'polypills' containing more than one active ingredient with different release profiles $[31,32]$. The polypill concept could arouse much interest in the development of personalised therapies in geriatric patients, as polypharmacy is a well-known issue in this patient group [33]. An example of a reported 3D printed polypill consists of three compartments designed as a captopril-loaded osmotic pump on the bottom and two separate top sections containing nifedipine and glipizide (Fig. 7) as part of an antihypertensive regime for diabetic patients [32].

A five API polypill was also developed, including three sustained release compartments containing pravastatin, atenolol and ramipril, and a top immediate release layer loaded
Fig. 7 Concept of a 3D printed polypill consisting of an osmotic pump coupled with a sustained release section and containing three different APIs. Picture reproduced with permission from [32]

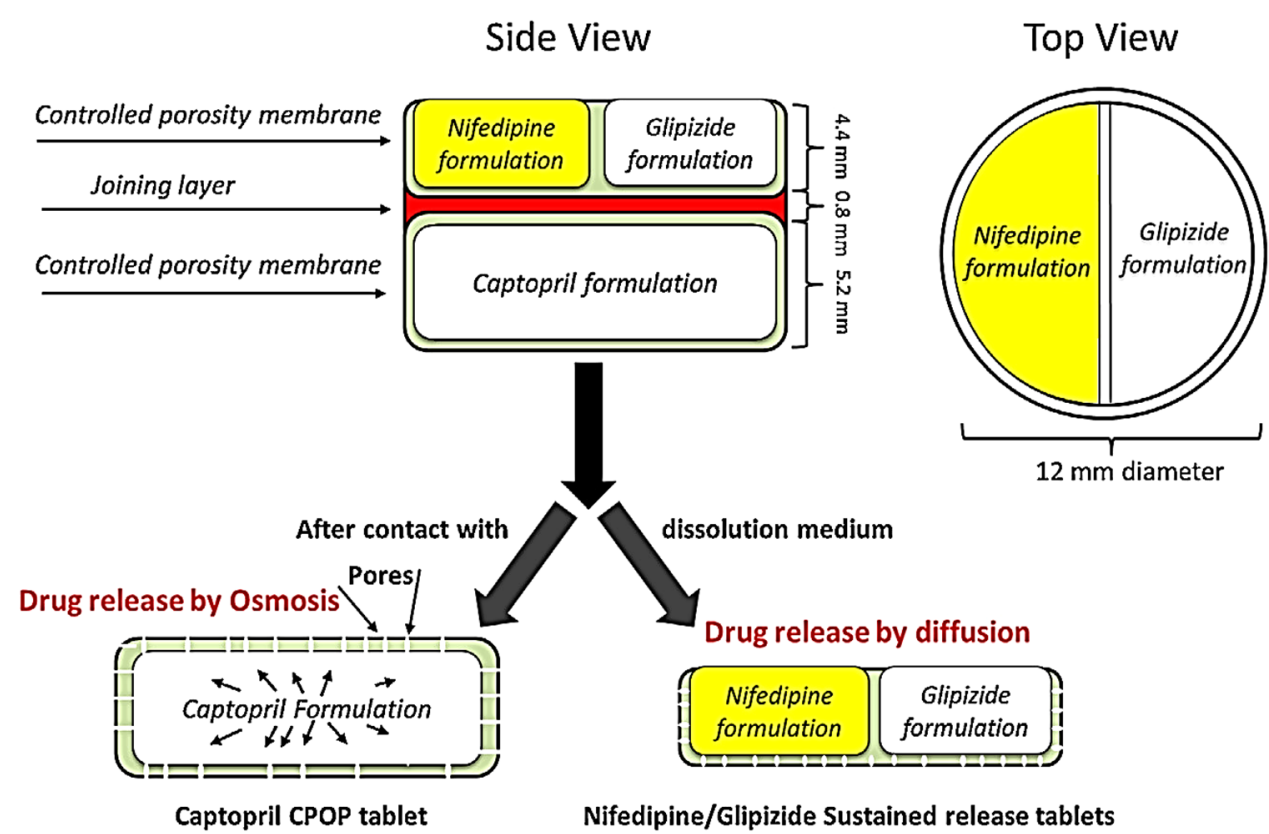


with aspirin and hydrochlorothiazide [31]. Compartments containing different APIs were separated using cellulose acetate to form a hydrophobic barrier. An important consideration when developing polypills regards the allocation of the APIs in the different layers; the top and the bottom layers will expose a larger area to the media and, therefore, drugs in those layers will be released faster. An effective strategy can be to include the less soluble APIs in the external layer, and the more soluble in the inner ones [34].

Despite their attractive features, PAM 3D printed tablets share a limitation related to the drying step required after production. In fact, the use of solvents such as ethanol, acetone and dimethyl sulfoxide is essential to produce pastes for PAM printing. Besides any toxicity concerns, which require the final product to be compliant with the ICH Guideline Q3C (R6) [35], an effective drying step is usually required when solvents are used, resulting in the possible degradation of thermolabile APIs when heat is used. Drying is also a time-consuming process $(24 \mathrm{~h}$ needed to dry a single tablet printed in $25 \mathrm{~min}$ ) [32]. Even when water was used to replace organic solvents, reducing drying time to $3 \mathrm{~h}$ at 80 ${ }^{\circ} \mathrm{C}$ [36], it should be considered that residual humidity in the final product could cause hydrolytic degradation of active ingredients and promote microbial growth [37].

\subsection{Fused deposition modelling}

Fused deposition modelling (FDM) is currently the most employed 3D printing technique in solid oral dosage form development; such a trend is due to the availability of inexpensive equipment that can even be coupled to conventional pharmaceutical processes such as hot melt extrusion (HME) and film coating [38]. Introduced in 1988 [39], FDM 3D printing became affordable to the general public in 2005 due to the RepRap (replicating rapid prototyper) project [40]. In FDM 3D printers, the feedstock material is a thermoplastic filament, which is melted and extruded through a heated nozzle; once extruded, the material returns to a solid, giving the object the desired geometry.
A characteristic parameter in FDM 3D printing is the infill percentage, defined as the printing density of a certain patterned structure inside the object (Fig. 8). Tuning the infill percentage has been explored to assess its impact on drug release and mechanical properties of tablets [41-43].

Early attempts of using FDM 3D printing to manufacture solid oral dosage forms reported polyvinyl alcohol (PVA) filaments loaded with model drugs by passive diffusion [44-46]. PVA is a water-soluble polymer having the advantage of being classified among Generally Recognised As Safe (GRAS) excipients [47] as well as a commercially available material for FDM 3D printing [48]; also, a novel PVA specifically designed for HME known as Parteck ${ }^{\circledR}$ MXP is available [49]. However, drug loading of PVA filaments by passive diffusion is low, therefore limiting the application to low dosages of active ingredients $[44,46]$.

Strategies to overcome such limitation are based on the application of hot melt extrusion (HME), a standard manufacturing process in the pharmaceutical industry, to produce printable filaments from pharmaceutical-grade excipients. Coupling HME with FDM 3D printing to improve drug loading of commercially available filaments such as PVA, does not affect the extrusion performances and, therefore, allows printing of devices with attractive design [15].

However, while PVA was initially used in proof of concept studies due to its established application in FDM, other pharmaceutical-grade polymers have been recently investigated to produce printable filaments via HME (Fig. 9) [50, 51]. For example, hydroxypropyl methylcellulose (HPMC) and Soluplus ${ }^{\circledR}$-based filaments were used to fabricate zeroorder drug release tablets [52], while Eudragit E PO was used to formulate filaments for immediate release tablets [53]. However, Sadia et al. (2016) reported a significant decrease in drug content after printing at a temperature of $135^{\circ} \mathrm{C}$, highlighting the main limitation of FDM 3D printing of medicines [53].

Temperatures up to $200{ }^{\circ} \mathrm{C}$ can be required in FDM, making this operation clearly unsuitable for thermolabile APIs $[45,49]$. To overcome this limitation, filaments extrudable at temperatures lower than $100{ }^{\circ} \mathrm{C}$ are required. However, FDM

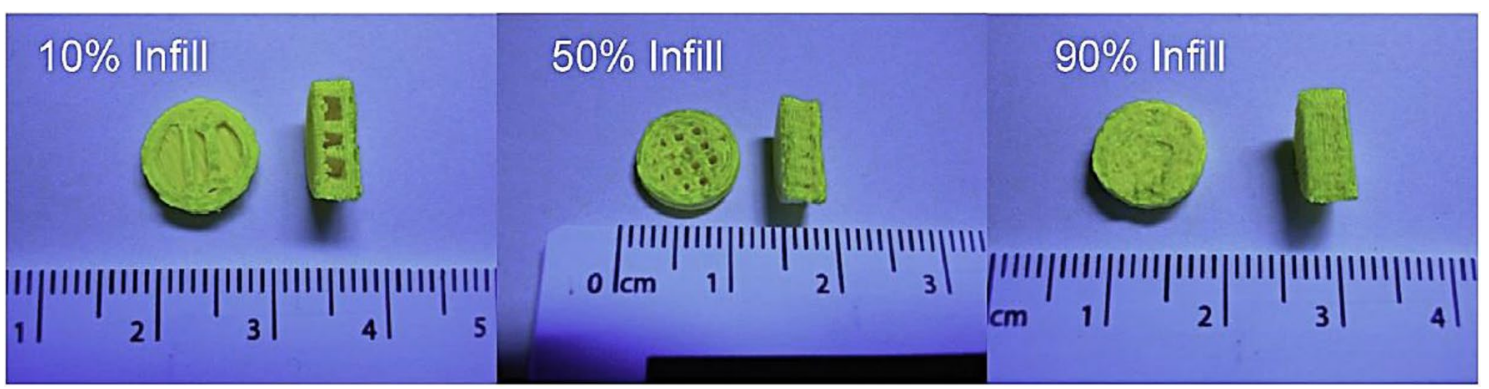

Fig. 8 Cross-sectional views of FDM 3D printed tablets with different infill percentage. Picture reproduced with permission from [44] 
Fig. 9 Diagram describing pharmaceutical grade polymers commonly employed in HME-FDM 3D printing. PVA is currently the most used polymer in solid oral dosage forms production

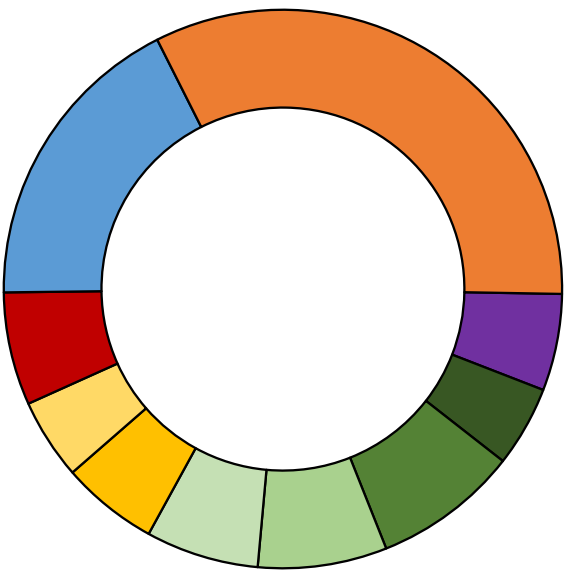

$\square$ Eudragit E PO

$\square$ Ethyl cellulose

$\square$ Hydroxypropyl cellulose

$\square$ Hydroxypropyl methylcellulose

$\square$ Hydroxypropyl methylcellulose acetate succinate

$\square$ Kollidon IR

$\square$ Kollidon VA 64

$\square$ Polyethylene glycol

$\square$ Polyvinyl alcohol

$\square$ Others is inherently designed to work with thermoplastic materials extruded at temperatures over $100{ }^{\circ} \mathrm{C}$.

Furthermore, the production of custom-made filaments introduces a more complex step to the relatively easy $3 \mathrm{D}$ printing process, requiring several optimisation studies to assess properties such as stiffness, toughness, melt viscosity and moisture uptake, usually altered by high drug loading $[52,54]$; filaments with unsuited mechanical properties can cause nozzle blockage and, therefore, printing failures [55].

\subsection{Selective laser sintering}

Selective laser sintering (SLS) works on similar principles as powder-bed inkjet 3D printing, with the difference that powder particles are sintered by a high-energy laser rather than bound by liquid solutions. The laser writes a specific pattern on the surface of the powder bed, corresponding to each layer. The immediate advantage of this is that organic solvents are not required, excluding the toxicity concerns and further drying steps.

The first application of SLS for pharmaceutical development consisted in the fabrication of polycaprolactonebased drug delivery devices [56]. Manufacturing of tablets by SLS using pharmaceutical polymers was recently described [57]; the presence of an additive absorbing light at the same wavelength as the laser $(445 \mathrm{~nm})$ was essential to observe particles sintering and Candurin $\AA^{\text {gold sheen }}$ was added to the polymer blend. Modulating laser scanning speed was also found as a useful strategy to alter tablets' porosity.

Such an approach was subsequently exploited to produce orally disintegrating tablets (ODTs) able to disintegrate within $4 \mathrm{~s}$ (Fig. 10), and dosage forms with a gyroid lattice geometry [58, 59]. The latter demonstrated the ability to tailor drug release by simply adjusting the tablet's design, thus avoiding modification in the formulation.
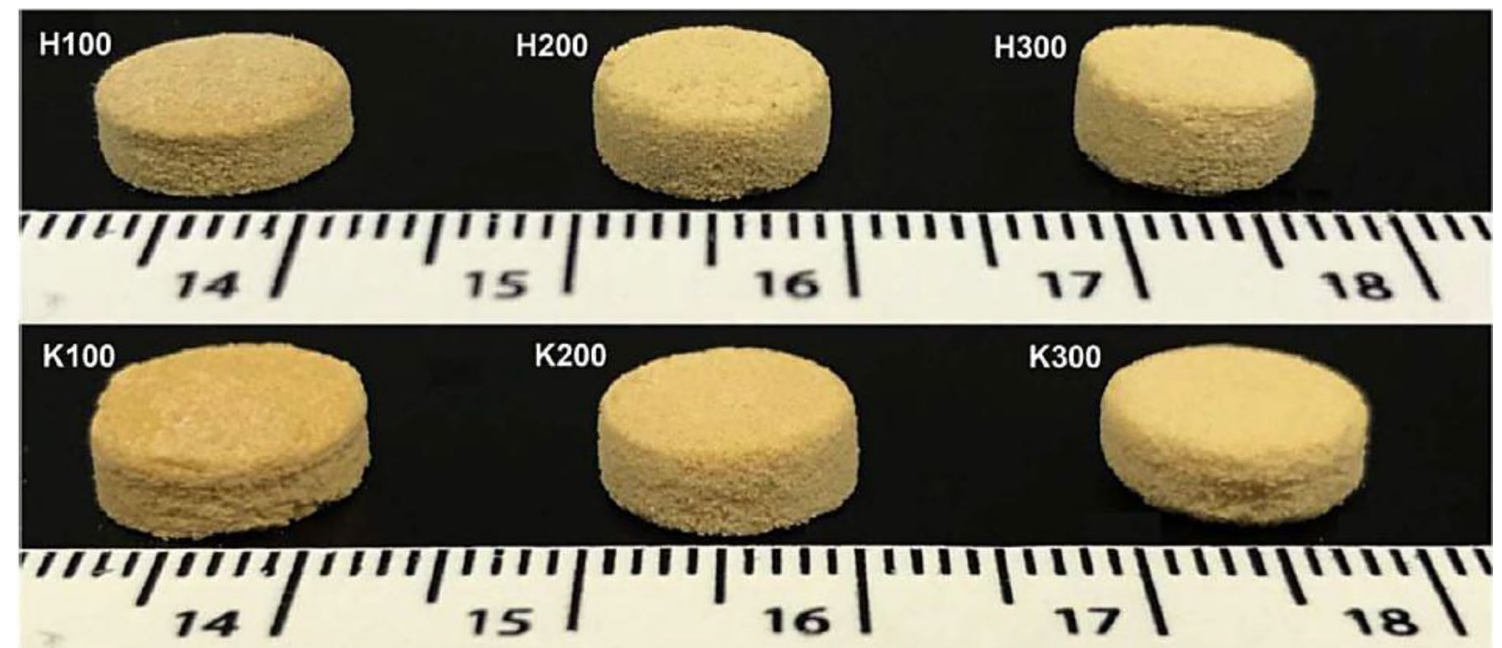

Fig. 10 Hydroxypropyl methylcellulose and Kollidon VA64 based tablets produced via SLS 3D printing. Picture reproduced with permission from [58] 
However, it should be considered that in all the studies carried out on SLS 3D printed dosage forms, paracetamol is the only drug used; therefore, there is no evidence that thermolabile APIs can be employed with this technique. In fact, when the laser hits a specific point of the powder bed, it increases the temperature. The local rise in temperature reaches a value between the melting point of the powdered material $\left(T_{\mathrm{m}}\right)$ and $T_{\mathrm{m}} / 2$, otherwise sintering would not occur. Despite no evidence of degradation of thermostable drug being observed [57], high heating developed during operation as well as the effect of high-power lasers may affect thermolabile APIs and further investigation is required. Also, sintering powdered materials makes tablets' appearance quite rough and that should be considered from a patient acceptability perspective.

\subsection{Stereolithography}

Stereolithography (SLA) is so far the least studied technique in solid oral dosage form development (Fig. 1); this is mainly attributable to the different working principles of SLA requiring specific materials currently not GRAS listed. In SLA, the fabrication of solid objects is based on a photopolymerisation process occurring when a focused laser beam hits a liquid photosensitive resin contained in a vat; a mirror galvanometer system directs the laser beam onto the liquid resin, drawing a pattern corresponding to each single layer.

Photopolymerisable resins employed in SLA are generally mixtures of reactive monomers and oligomers that cross-link when exposed to light of a certain wavelength in the presence of a photoinitiator. Important factors that

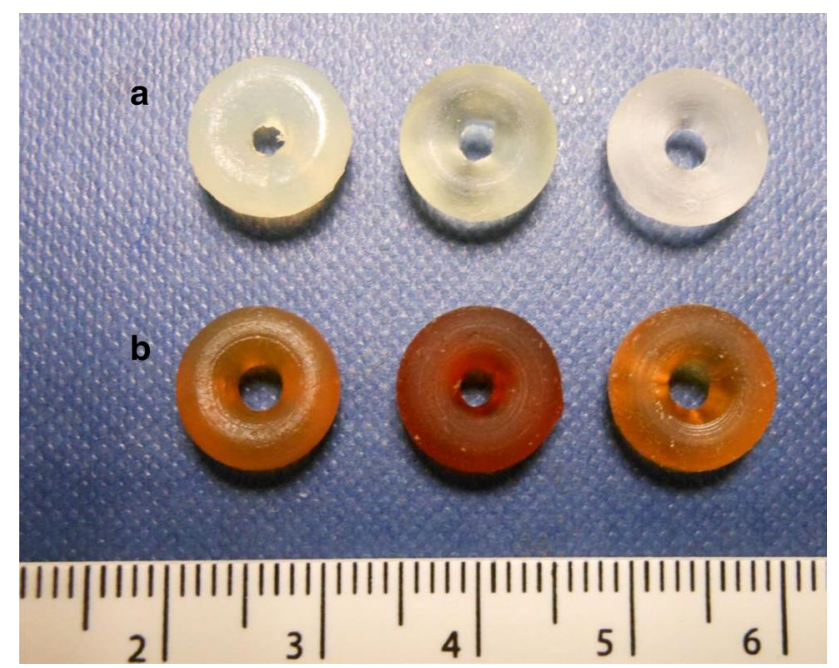

Fig. 11 Torus-shaped tablets printed via SLA. Tablets were formulated using PEGDA 700 and PEG300 at different ratio; a paracetamol and $\mathbf{b}$ 4-ASA were loaded in the liquid resin and then printed. Picture reproduced with permission from [61] can be controlled in SLA are laser power $(\mathrm{mW})$ and laser speed $(\mathrm{mm} / \mathrm{s})$; they can be used to tune the properties of the printed object or optimised to avoid potential degradation of photolabile APIs.

SLA is a very intriguing technique, as it does not share some of the limitations of the other 3D printing techniques. Since powders are not used to directly feed the machine, flow properties do not represent a concern; SLA printed structures do not present porous structures, therefore physical properties are advantageous; heating is not required during operation and degradation of APIs might be related only to the effect of the UV laser in the case of photolabile drugs being used. Furthermore, SLA offers the highest printing resolution among other technologies, an important feature to consider when developing complex geometries. In fact, as the resolution along the $\mathrm{X}-\mathrm{Y}$ axis is dictated by the width of the laser beam, resolution up to $70 \mu \mathrm{m}$ can be achieved in recent commercial printers [60]; resolution on the $Z$ axis (vertical resolution) is instead controlled by the depth of curing that can be adjusted by varying laser settings and composition of the resin [48].

On the other hand, resins used in SLA are highly reactive and the presence of unreacted compounds, coupled with the nature itself of the free radical polymerisation process, is a concern holding back the development of this technique in the pharmaceutical field.

The first application of SLA in the formulation of 3D printed tablets was described by Wang et al. (2016), who produced dosage forms in a torus geometry using a photosensitive resin consisting of polyethylene glycol diacrylate 700 (PEGDA 700) and diphenyl(2,4,6-trimethylbenzoyl) phosphineoxide (TPO) as a photoinitiator (Fig. 11) [61]. Polyethylene glycol 300 was found to increase drug release and it is likely that it reduces the degree of cross-linking of PEGDA. 4-aminosalicylic acid (4-ASA) and paracetamol were used as model drugs and degradation was not observed.

SLA was also investigated to fabricate drug-loaded hydrogels, and a photoinitiator system based on riboflavin and triethanolamine was tested as a biocompatible alternative to TPO [62]. The feasibility of SLA in printing complex shapes encourages the study of the effect of dosage form geometries on drug release profiles. In fact, drug release profiles could be affected with unpredictable effects by modifying size and/ or shape of tablets, and preliminary evidence shows that the surface area/volume ratio $(\mathrm{SA} / \mathrm{V})$ is the parameter with the highest influence on release profiles [63].

Although the reported works describe the validity of SLA to produce solid oral dosage forms, there remain significant limitations to be addressed, in particular the toxicity of photopolymer resins coupled with the low availability of safe photopolymerisable compounds and novel excipients. To the best of our knowledge, no data are currently available on the cytotoxicity tablets produced using SLA, and studies in the 
field are therefore required to confirm the good potential of the technique. Further, screening and evaluation of monomers, oligomers and photoinitiators could enlarge the pool of materials suitable for SLA tablet manufacture, with specific enthusiasm on the development of novel excipients.

\section{Areas of application of 3D printed drug products}

\subsection{Personalised pharmacological treatments}

The rationale behind 3D printing pharmaceuticals relies on providing an adaptable platform to produce and dispense tailored drug products [4]. 3D printing can serve the purpose by manufacturing dosage forms with adjustable properties, e.g. bespoke drug dosages and/or release profiles [54, 64]. Personalising medicinal products also encompasses features such as size, shape, texture, colour and flavour of oral dosage forms, all of which may influence patients' acceptability criteria [65-67]. Providing treatments able to satisfy individual needs is considered a goal of medicine in the near future; continuous progresses in pharmacogenomics and bioinformatics will help to predict patients' responses to medicinal products, improving therapeutic outcomes with benefits to both patients and the economy [3]. From a clinical perspective, personalisation of pharmacotherapies shows higher importance where drugs with a narrow therapeutic index (NTI) are used. An important application could be represented by oral anticoagulants [68-70]; coumarin-based regimens are known to be poorly managed because of the many interactions with food and other drugs, requiring the continuous INR profile monitoring and dose titration [71]; frequent or even daily dose adjustments could be easily made by modifying the digital design used to $3 \mathrm{D}$ print a dosage form, with no need to alter the formulation.

Personalising medicine also means providing an alternative drug product to those patients with known allergies to commonly used excipients, such as lactose [72]. A commercial alternative is not always available, and some individuals may suffer serious side effects when exposed to the allergen.

Moreover, personalising medicine could address polypharmacy issues, especially for elderly patients [33]. More active ingredients can be combined in the bespoke 'polypills', reducing the daily amount of tablet intake, improving patient compliance and therapy adherence. For instance, specific formulations could be designed for the treatment of those diseases requiring a complex therapeutic regimen, such as diabetes [7, 31, 32].

Hospital pharmacies have a primary role in dispensing such personalised pharmaceutical products. The future for 3D printing in this setting could see a complete cycle of diagnosis through to treatment all at the point of care. A possible example would see a patient in hospital undertaking a test which elucidates the individuals' personal profile, this may be based on genetics or molecular composition of fluid samples. Based on this, the patient would receive a bespoke prescription which precisely addresses the patients' needs. This precise and personalised prescription would then be translated into a bespoke dosage form via an adaptable and dynamic dosage form design and a suitable 3D printer. With current technology, the time to produce quality $3 \mathrm{D}$ printed tablets is short, typically $1-2 \mathrm{~h}$ at most and further technological advances within the printers and materials for tablet production promises to reduce this further. When coupled with parallel advances in testing, diagnosis and data processing informing personalised medicine, this approach promises to deliver truly personalised medicine.

\subsection{On-demand production of drug products}

Due to the highly expensive equipment and the spacious facilities required, setting up a conventional pharmaceutical production site is limited to industrial plants, where large batches are daily manufactured and transported remotely.

Despite its established effectiveness, such a system is not without limitations: for example, failure to supply medicines to remote areas of the world and in developing countries, due to difficulties that can be encountered in long-distance transport, natural disasters or conflicts [73].

Such limitations can potentially be overcome with the establishment of novel technologies like 3D printing, which is emerging as a promising tool for the on-demand production of oral dosage forms [74]. Low cost, compact size and almost unlimited production flexibility make 3D printing a frontrunner technology capable of being employed in various scenarios where the same machine can be used to fabricate personalised medicines, prosthesis, equipment and even to synthesise APIs [75-77]. Such potential could be exploited to accelerate clinical trials, where it is necessary to design a wide range of dosage forms with different dosages and release profiles to evaluate novel API efficacy [4]. This application is likely the most suitable to meet the pharmaceutical industry needs through 3D printing.

Further, an ambitious application of pharmaceutical AM relies on future space exploration, since AM has already been experimented on the International Space Station to produce tools on-site [78]. It is, therefore, reasonable to imagine that long space journeys involving human crews will require versatile pharmaceutical workstations able to supply medicines and medical devices with unique characteristics. 


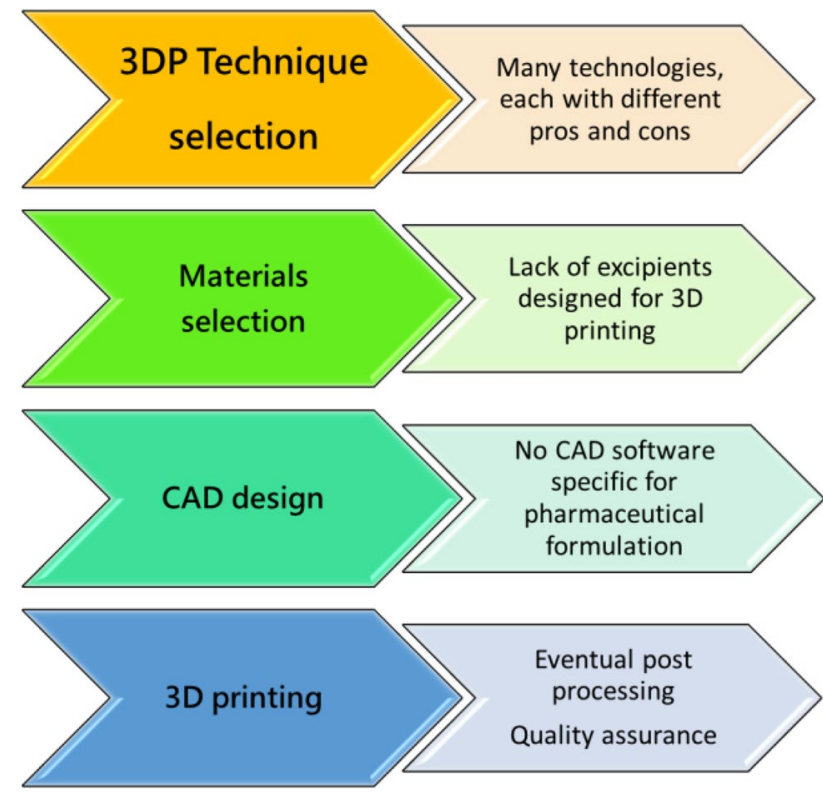

Fig. 12 Stages in 3D printing and relative pharmaceutical challenges

\section{The regulatory framework}

At the present time, no regulatory pathway for bespoke 3D printed oral dosage forms is established. Although Aprecia Pharmaceuticals demonstrated how 3D printing can be a realistic method of manufacture, representing a breakthrough point for the development of the technology, Spritam ${ }^{\circledR}$ is not considered as a personalised product, as it is only available in four dosages. Therefore, even though $3 \mathrm{D}$ printing can meet the requirements for pharmaceuticals manufacture, it seems that approval can only be granted when the process is intended for mass production. However, current research on $3 \mathrm{D}$ printing in pharmaceutics is mainly focused on personalised medicine, targeting the fabrication of dosage forms with properties varying from batch to batch. This is inherently in conflict with the requirements of pharmaceutical production processes, for which the inter-batch variability must be guaranteed to a minimum. Given such limitations caused by legislation, it will likely take time for 3D printing approaches to be awarded widespread regulatory approval, as changes in regulation will likely be implemented incrementally. For the present, it is likely that the manufacture of 3D printed medicines will be limited to fixed dose units as has proven successful in the case of Spritam ${ }^{\circledR}$ [79].

Considering the key steps in 3D printing, weak points in validating the process rely on the lack of 3D printers specifically designed for pharmaceutical applications, on the material selection-in case novel excipients are needed-and on the unavailability of advanced CAD software, able to adapt tablet design to the desired therapeutical properties (Fig. 12).
A further observation regarding the on-demand manufacturing of a wide range of medicines, for example in hospital pharmacies, highlights the need of appropriate and accurate analytical procedures to ensure final product quality; process analytical technologies such as near infrared spectroscopy and Raman confocal microscopy were recently found as valid techniques for the intended purpose [80].

Although it is necessary for the scientific community to build a solid knowledge background to understand and improve the whole manufacturing process from dosage form design to the relative quality control, it is essential that regulatory agencies issue guidelines that can promote development and safe application of 3D printing technology in clinical scenarios.

\section{Conclusion}

Several formulation approaches to three-dimensional printing of solid oral dosage forms have been described and discussed. Selecting which 3D printing technique is employed to fabricate drug products requires careful consideration of the respective advantages and disadvantages of each technique, as well as the final product's desired characteristics. For example, fused deposition modelling is emerging as a frontrunner technology due to its compatibility with established pharmaceutical excipients and processes, but it should not be considered in the development of thermolabile API-based products. Another example is stereolithography, still in its infancy due to its toxicity concerns; however, its high-resolution power could be exploited to study the relationship between complex design and dosage form properties. According to this, an important step toward the clinical application of 3D printing is to identify the advantages of the different technologies with respect to the production of medicinal products and merge them in a $3 \mathrm{D}$ printer specifically designed for pharmaceutical manufacture.

To conclude, we believe that the path to the medical application of 3D printing has now been traced and it is, therefore, possible to envision a future with smart, accessible and personalised medicine, where novel technologies will not replace conventional large-scale manufacturing, but rather meet the unmet needs of contemporary medicine.

Open Access This article is licensed under a Creative Commons Attribution 4.0 International License, which permits use, sharing, adaptation, distribution and reproduction in any medium or format, as long as you give appropriate credit to the original author(s) and the source, provide a link to the Creative Commons licence, and indicate if changes were made. The images or other third party material in this article are included in the article's Creative Commons licence, unless indicated otherwise in a credit line to the material. If material is not included in the article's Creative Commons licence and your intended use is not permitted by statutory regulation or exceeds the permitted use, you will 
need to obtain permission directly from the copyright holder. To view a copy of this licence, visit http://creativecommons.org/licenses/by/4.0/.

\section{References}

1. Augsburger L, Hoag S (2008) Pharmaceutical Dosage Forms Tablets, 3rd ed

2. Sastry S, Nyshadham J, Fix J (2000) Recent technological advances in oral drug delivery - a review. Pharm Sci Technolo Today 3(4):138-145

3. Graham E (2016) "Improving outcomes through personalised medicine." NHS Engl 6-10

4. Trenfield SJ, Awad A, Goyanes A, Gaisford S, Basit AW (2018) 3D Printing Pharmaceuticals: Drug Development to Frontline Care. Trends Pharmacol Sci 39(5):440-451

5. Pritchard DE, Moeckel F, Villa MS, Housman LT, McCarty CA, McLeod HL (2017) Strategies for integrating personalized medicine into healthcare practice. Per Med 14(2):141-152

6. Goole J, Amighi K (2016) 3D printing in pharmaceutics: A new tool for designing customized drug delivery systems. Int J Pharm 499(1-2):376-394

7. Pereira BC, Isreb A, Forbes RT, Dores F, Habashy R (2019) European journal of pharmaceutics and biopharmaceutics ' temporary plasticiser': A novel solution to fabricate 3D printed patient- centred cardiovascular ' Polypill ' architectures. Eur J Pharm Biopharm 135:94-103

8. Tagami $\mathrm{T}$ et al (2018) Defined drug release from 3D-printed composite tablets consisting of drug-loaded polyvinylalcohol and a water-soluble or water-insoluble polymer filler. Int J Pharm 543(1-2):361-367

9. Okwuosa TC, Soares C, Gollwitzer V, Habashy R, Timmins P, Alhnan MA (2018) On demand manufacturing of patient-specific liquid capsules via co-ordinated $3 \mathrm{D}$ printing and liquid dispensing. Eur J Pharm Sci 118(February):134-143

10. Sadia M, Arafat B, Ahmed W, Forbes RT, Alhnan MA (2018) Channelled tablets: An innovative approach to accelerating drug release from 3D printed tablets. J Control Release 269:355-363

11. Arafat B et al (2018) Tablet fragmentation without a disintegrant: A novel design approach for accelerating disintegration and drug release from 3D printed cellulosic tablets. Eur J Pharm Sci 118(March):191-199

12. Chai $X$ et al (2017) Fused deposition modeling (FDM) 3D printed tablets for intragastric floating delivery of domperidone. Sci Rep 7(1):1-9

13. Goyanes A, Fina F, Martorana A, Sedough D, Gaisford S, Basit AW (2017) Development of modified release 3D printed tablets (printlets) with pharmaceutical excipients using additive manufacturing. Int J Pharm 527(1-2):21-30

14. Nukala PK, Palekar S, Patki M, Patel K (2019) Abuse deterrent immediate release egg-shaped tablet (egglets) using 3D printing technology: quality by design to optimize drug release and extraction. AAPS PharmSciTech 20(2):80

15. Goyanes A et al (2015) 3D Printing of medicines: engineering novel oral devices with unique design and drug release characteristics. Mol Pharm 12(11):4077-4084

16. Infanger S, Haemmerli A, Iliev S, Baier A, Stoyanov E, Quodbach J (2019) Powder bed 3D-printing of highly loaded drug delivery devices with hydroxypropyl cellulose as solid binder. Int J Pharm 555:198-206

17. Wu BM, Borland SW, Giordano RA, Cima LG, Sachs EM, Cima MJ (1996) Solid free-form fabrication of drug delivery devices. J Control Release 40(1-2):77-87
18. Katstra W, Palazzolo R, Rowe C, Giritlioglu B, Teung P, Cima M (2000) Oral dosage forms fabricated by Three Dimensional Printing ${ }^{\mathrm{TM}}$. J Control Release 66(1):1-9

19. Rowe C, Katstra W, Palazzolo R, Giritlioglu B, Teung P, Cima M (2000) Multimechanism oral dosage forms fabricated by three dimensional printing ${ }^{\mathrm{TM}}$. J Control Release 66(1):11-17

20. Yu DG, Yang XL, Huang WD, Liu J, Wang YG, Xu H (2007) Tablets With Material Gradients Fabricated by Three-Dimensional Printing. J Pharm Sci 96(9):2446-2456

21. Gokhale A (2014) Achieving zero-order release kinetics using multi-step diffusion-based drug delivery. Pharm Technol Eur 26(5):38-42

22. Paarakh MP, Jose PA, Setty CM, Peter GV (2018) Release kinetics-concepts and applications. Int J Pharm Res Technol 10(1):1-9

23. Yu DG, Branford-White C, Ma ZH, Zhu LM, Li XY, Yang XL (2009) Novel drug delivery devices for providing linear release profiles fabricated by 3DP. Int J Pharm 370(1-2):160-166

24. Yu D-G, Shen X-X, Branford-White C, Zhu L-M, White K, Yang XL (2009) Novel oral fast-disintegrating drug delivery devices with predefined inner structure fabricated by three-dimensional printing. J Pharm Pharmacol 61(3):323-329

25. Fitzgerald S (2015) FDA Approves First 3D-printed epilepsy drug experts assess the benefits and caveats. Neurol Today 15(18):26-27

26. Boudriau S, Hanzel C, Massicotte J, Sayegh L, Wang J, Lefebvre M (2016) Randomized comparative bioavailability of a novel three-dimensional printed fast-melt formulation of levetiracetam following the administration of a single 1000-mg dose to healthy human volunteers under fasting and fed conditions. Drugs R D 16(2):229-238

27. Prescott J, Barnum R (2000) On powder flowability. Pharm Technol 24(October):60-84

28. British Pharmacopoeia / Appendix XVII G, Friability of Uncoated Tablets, Ph Eur method (2.9.7); 2015

29. Malone E, Lipson H (2007) Fab@Home: The personal desktop fabricator kit. Rapid Prototyp J 13(4):245-255

30. Khaled SA, Burley JC, Alexander MR, Roberts CJ (2014) Desktop 3D printing of controlled release pharmaceutical bilayer tablets. Int J Pharm 461(1-2): 105-111

31. Khaled SA, Burley JC, Alexander MR, Yang J, Roberts CJ (2015) $3 \mathrm{D}$ printing of five-in-one dose combination polypill with defined immediate and sustained release profiles. J Control Release 217:308-314

32. Khaled SA, Burley JC, Alexander MR, Yang J, Roberts CJ (2015) $3 \mathrm{D}$ printing of tablets containing multiple drugs with defined release profiles. Int J Pharm 494(2):643-650

33. Hajjar ER, Cafiero AC, Hanlon JT (2007) Polypharmacy in elderly patients. Am J Geriatr Pharmacother 5(4):345-351

34. Robles-Martinez P, Xu X, Trenfield SJ, Awad A, Goyanes A, Telford R, Basit A, Gaisford S (2019) 3D printing of a multi-layered polypill containing six drugs using a novel stereolithographic method. Pharmaceutics 11(6):274

35. ICH, I. Q3C (R6) Impurities: Guideline for Residual Solvents. 2016

36. Khaled SA et al (2018) 3D extrusion printing of high drug loading immediate release paracetamol tablets. Int $\mathbf{J}$ Pharm 538(1-2):223-230

37. Carstensen JT (1988) Effect of moisture on the stability of solid dosage forms. Drug Dev Ind Pharm 14(14):1927-1969

38. Goyanes A et al (2015) Fabrication of controlled-release budesonide tablets via desktop (FDM) 3D printing. Int J Pharm 496(2):414-420

39. Crump SS (1992) U.S. Patent No. 5,121,329. U.S. Patent and Trademark Office, Washington, DC

40. Jones R et al (2011) RepRap - the replicating rapid prototyper. Robotica 29(1):177-191 
41. Tagami T, Fukushige K, Ogawa E, Hayashi N, Ozeki T (2017) 3D Printing factors important for the fabrication of polyvinylalcohol filament-based tablets. Biol Pharm Bull 40(3):357-364

42. Yang Y, Wang H, Li H, Ou Z, Yang G (2018) 3D printed tablets with internal scaffold structure using ethyl cellulose to achieve sustained ibuprofen release. Eur J Pharm Sci 115:11-18

43. Fuenmayor E et al (2019) Comparison of fused- fi lament fabrication to direct compression and injection molding in the manufacture of oral tablets. Int J Pharm 558(January):328-340

44. Goyanes A, Buanz ABM, Basit AW, Gaisford S (2014) Fusedfilament 3D printing (3DP) for fabrication of tablets. Int J Pharm 476(1):88-92

45. Goyanes A, Buanz ABM, Hatton GB, Gaisford S, Basit AW (2015) 3D printing of modified-release aminosalicylate (4-ASA and 5-ASA) tablets. Eur J Pharm Biopharm 89:157-162

46. Skowyra J, Pietrzak K, Alhnan MA (2015) Fabrication of extended-release patient-tailored prednisolone tablets via fused deposition modelling (FDM) 3D printing. Eur J Pharm Sci 68:11-17

47. Hallagan JB (2003) Polyvinyl alcohol notification. U.S. Food \& Drug Administration. http://wayback.archive-it.org/7993/20171 031052944/https://www.fda.gov/downloads/Food/Ingredient sPackagingLabeling/GRAS/NoticeInventory/UCM267720.pdf. Accessed 17 Mar 2020

48. Ligon SC, Liska R, Stampfl J, Gurr M, Mülhaupt R (2017) Polymers for 3D printing and customized additive manufacturing. Chem Rev 117(15):10212-10290

49. Nukala PK, Palekar S, Solanki N, Fu Y, Patki M, Ali A (2019) Investigating the application of fused deposition modeling 3D printing pattern in preparation of patient-tailored dosage forms. $\mathrm{J}$ 3D Print Med. https://doi.org/10.2217/3dp-2018-0028

50. Solanki NG, Tahsin M, Shah AV, Serajuddin ATM (2018) Formulation of 3D printed tablet for rapid drug release by fused deposition modeling: screening polymers for drug release, drug-polymer miscibility and printability. J Pharm Sci 107(1):390-401

51. Melocchi A, Parietti F, Maroni A, Foppoli A, Gazzaniga A, Zema L (2016) Hot-melt extruded filaments based on pharmaceutical grade polymers for 3D printing by fused deposition modeling. Int J Pharm 509(1-2):255-263

52. Zhang J, Feng X, Patil H, Tiwari RV, Repka MA (2017) Coupling $3 \mathrm{D}$ printing with hot-melt extrusion to produce controlled-release tablets. Int J Pharm 519(1-2):186-197

53. Sadia $M$ et al (2016) Adaptation of pharmaceutical excipients to FDM 3D printing for the fabrication of patient-tailored immediate release tablets. Int J Pharm 513(1-2):659-668

54. Öblom $\mathrm{H}$ et al (2019) 3D-printed isoniazid tablets for the treatment and prevention of tuberculosis-personalized dosing and drug release. AAPS PharmSciTech 20:52. https://doi.org/10.1208/ s12249-018-1233-7

55. Nasereddin JM, Wellner N, Alhijjaj M, Belton P, Qi S (2018) Development of a simple mechanical screening method for predicting the feedability of a pharmaceutical FDM 3D printing filament. Pharm Res 35(8):151

56. Leong KF, Wiria FE, Chua CK, Li SH (2007) Characterization of a poly- $\varepsilon$-caprolactone polymeric drug delivery device built by selective laser sintering. Biomed Mater Eng 17(3):147-157

57. Fina F, Goyanes A, Gaisford S, Basit AW (2017) Selective laser sintering (SLS) 3D printing of medicines. Int J Pharm 529(1-2):285-293

58. Fina F, Madla CM, Goyanes A, Zhang J, Gaisford S, Basit AW (2018) Fabricating 3D printed orally disintegrating printlets using selective laser sintering. Int J Pharm 541(1-2):101-107

59. Fina $\mathrm{F}$ et al (2018) 3D printing of drug-loaded gyroid lattices using selective laser sintering. Int J Pharm 547(1-2):44-52

60. Basso A, Mendez Ribo M, Danielak AH, Yang B, Kjeldsteen P, Valler P, Zhang Y (2019) 3d printed mold for powder injection molding process. In: Bernard A, Leach RK, Pedersen DB, Taylor JS (eds) Proceedings of the Joint Special Interest Group meeting between euspen and ASPE Advancing Precision in Additive Manufacturing (2019) The European Society for Precision Engineering and Nanotechnology, pp 71-74. https://peopoly.net/produ cts/moai

61. Wang J, Goyanes A, Gaisford S, Basit AW (2016) Stereolithographic (SLA) 3D printing of oral modified-release dosage forms. Int J Pharm 503(1-2):207-212

62. Martinez PR, Goyanes A, Basit AW, Gaisford S (2017) Fabrication of drug-loaded hydrogels with stereolithographic 3D printing. Int J Pharm 532(1):313-317

63. Martinez PR, Goyanes A, Basit AW, Gaisford S (2018) Influence of geometry on the drug release proiles of stereolithographic (SLA) 3D-printed tablets. AAPS PharmSciTech 19(8):3355-3361

64. Fina F, Goyanes A, Madla CM, Awad A, Tren SJ (2018) 3D printing of drug-loaded gyroid lattices using selective laser sintering. Int J Pharm 547(1-2):44-52

65. Yoder S, Rajabi J, Miller C, Oza K (2014) Physical appearance preferences for oral solid dosage formulations. AAPS Poster 2018:1-4

66. Goyanes A, Scarpa M, Kamlow M, Gaisford S, Basit AW, Orlu M (2017) Patient acceptability of 3D printed medicines. Int J Pharm 530(1-2):71-78

67. Scoutaris N, Ross SA, Douroumis D (2018) 3D Printed 'Starmix' drug loaded dosage forms for paediatric applications. Pharm Res 35(2):1-11

68. Epstein RS et al (2010) Warfarin genotyping reduces hospitalization rates. Results from the MM-WES (Medco-Mayo Warfarin Effectiveness Study). J Am Coll Cardiol 55(25):2804-2812

69. Wadelius $\mathrm{M}$ et al (2005) Common VKORC1 and GGCX polymorphisms associated with warfarin dose. Pharmacogen $\mathrm{J}$ 5(4):262-270

70. Arafat B, Qinna N, Cieszynska M, Forbes RT, Alhnan MA (2018) Tailored on demand anti-coagulant dosing: an in vitro and in vivo evaluation of 3D printed purpose-designed oral dosage forms. Eur J Pharma Biopharm 128:282-289

71. Holbrook AM et al (2005) Systematic overview of warfarin and its drug and food interactions. Arch Intern Med 165(10):1095

72. Ursino MG, Poluzzi E, Caramella C, De Ponti F (2011) Excipients in medicinal products used in gastroenterology as a possible cause of side effects. Regul Toxicol Pharmacol 60(1):93-105

73. Saripalle S, Maker H, Bush A and Lundman N (2016) 3D printing for disaster preparedness: making life-saving supplies on-site, ondemand, on-time. IEEE Global Humanitarian Technology Conference (GHTC), Seattle, WA, pp. 205-208

74. El Aita I, Breitkreutz J, Quodbach J (2019) On-demand manufacturing of immediate release levetiracetam tablets using pressureassisted microsyringe printing. Eur J Pharm Biopharm 134:29-36

75. Yu AW, Khan M (2015) On-demand three-dimensional printing of surgical supplies in conflict zones. J Trauma Acute Care Surg 78(1):201-203

76. Kondor $\mathrm{S}$ et al (2013) On demand additive manufacturing of a basic surgical kit. J Med Device 7(3):030916

77. Kitson PJ et al (2018) Digitization of multistep organic synthesis in reactionware for on-demand pharmaceuticals. Science 359(6373):314-319

78. Harbaugh J (2017) Space station 3-D printer builds ratchet wrench to complete first phase of operations. NASA. www.nasa.gov/missi on_pages/station/research/news/3Dratchet_wrench. Accessed 28 Sept 2018

79. Brandessence Market Research Company Pvt Ltd (2019) 3D Printed Drugs Market 2019 Top Key Players are 3D Printing Systems, Aprecia Pharmaceuticals, Hewlett Packard Enterprise, Hewlett Packard Enterprise, GLAXOSMITHKLINE PLC and Forecast to 2025. Medgadget, Inc. https://www.medgadget. 
com/2019/09/3d-printed-drugs-market-2019-top-key-playersare-3d-printing-systems-aprecia-pharmaceuticals-hewlett-packa rd-enterprise-hewlett-packard-enterprise-glaxosmithkline-plcand-forecast-to-2025.html. Accessed 17 Mar 2020

80. Trenield SJ, Goyanes A, Telford R, Wilsdon D, Rowland M (2018)

3D printed drug products: Non-destructive dose veriication using a rapid point-and-shoot approach. Int J Pharm 549(1-2):283-292
Publisher's Note Springer Nature remains neutral with regard to jurisdictional claims in published maps and institutional affiliations. 